\title{
Low-Energy X-Ray Standards from Hydrogenlike Pionic Atoms
}

\author{
D. F. Anagnostopoulos, ${ }^{1}$ D. Gotta,${ }^{2}$ P. Indelicato,${ }^{3, *}$ and L. M. Simons ${ }^{4}$ \\ ${ }^{1}$ Department of Materials Science and Engineering, University of Ioannina, GR-45110 Ioannina, Greece \\ ${ }^{2}$ Institut für Kernphysik, Forschungszentrum Jülich, D-52425 Jülich, Germany \\ ${ }^{3}$ Laboratoire Kastler Brossel, École Normale Supérieure et Université Pierre et Marie Curie, Case 74, 4 place Jussieu, \\ 75005 Paris, France \\ ${ }^{4}$ Paul-Scherrer-Institut (PSI), CH 5232-Villigen, Switzerland
}

(Received 26 June 2002; published 10 December 2003)

\begin{abstract}
We demonstrate the first step of a complete program, which consists in establishing an x-ray energy standard scale with the use of few-body atoms, in the few $\mathrm{keV}$ range. Light pionic and muonic atoms as well as one and two-electron ions from electron-cyclotron ion sources are used. The transition energies are calculable from quantum-electrodynamics, meaning that only a very limited subset need be measured and compared with theory, while providing a large number of standard lines. Here we show that circular transitions in pionic neon atoms, completely stripped from their electrons, reveal spectral lines which are narrow, symmetric, and well reproducible. We use these lines for the energy determination of transition energies in complex electronic systems, like the $\mathrm{K} \alpha_{1,2}$ transitions in metallic Ti, which may serve as secondary standard.
\end{abstract}

DOI: 10.1103/PhysRevLett.91.240801

Accurate (below $1 \mathrm{ppm}$ ) and reproducible $\mathrm{x}$-ray wavelength standards with reasonably dense set of lines would be very valuable for the most widespread application of $x$ rays: the determination of crystal lattice parameters with diffractometric methods (see, e.g., Ref. [1] and references therein). Other practical applications are found, such as the energy calibration of synchrotron radiation beams, monochromators and spectrometers, and the determination of the response function of $\mathrm{x}$-ray spectrometers and diffractometers. X-ray standards can thus be useful in many areas of modern science such as crystallography, solid state, molecular, atomic and particle physics, chemistry, and biochemistry.

A recent experiment used the ${ }^{57} \mathrm{Fe}$ Mössbauer radiation, excited by synchrotron radiation, improved the energy (wavelength) standard for the energy region of $14 \mathrm{keV}$ by 2 orders of magnitude in accuracy from 10 to $0.2 \mathrm{ppm}$ [2]. This attempt, while very promising, is very difficult to extend to lower energies, where electron conversion would dramatically reduce the nuclear fluorescence. In the absence of an appropriate excitation source, such as synchroton radiation, this would require unrealistically high source activities with the additional requirement of a sufficiently long lifetime of the parent isotope. In addition self-absorption of low-energy $\mathrm{x}$ rays in the source is very strong. The $\mathrm{x}$ rays can thus only originate from the surface layer, which leads to an upper bound to the maximum effective activity that can be reached by increasing the amount radioactive material. Finally, all these transitions are orders of magnitude narrower than crystal spectrometers resolution. For some applications the extreme narrowness of $\gamma$ lines is of no use while limiting severely their intensity.

The most widely used x-ray energy standards, at the present time, are made by exciting innershell transitions
PACS numbers: 06.20.Fn, 07.85.Nc, 32.30.Rj, 36.10.-k

in atoms with either electrons or photons. In a number of cases their energies are given with precision close to $1 \mathrm{ppm}$ [3], which does not necessarily mean that these standards can be used to such an accuracy. For $\mathrm{x}$ rays originating from innershell transitions in multielectron systems, the center of gravity of the line cannot be attributed unambiguously to a physical transition. Shake-off processes (which create additional vacancies) and open outer shells lead to numerous satellite transitions very close in energy to the diagram line, which cannot be resolved and produce asymmetric line shapes. Moreover, the line shape of transitions in multielectronic systems depends also on the excitation mechanism used to create the innershell vacancies. For example the evolution of the $\mathrm{K}\left(1 s^{-1}\right)$ argon spectrum has been studied as function of excitation energy $[4,5]$, and dramatic qualitative changes were observed. The chemical environment of the atom also plays a strong role as can be seen from the comparison between solids, metallic vapors, and theoretical x-ray absorption edges energies [3,6,7]. This problem also affects transition energies, particularly when they involve $\mathrm{M}_{2,3}$ and $\mathrm{N}_{2,3}$ shells.

An inherent problem with current standard $\mathrm{x}$-ray lines is their natural width, which is typically more than 10 times larger than the resolution of the best $\mathrm{x}$-ray spectrometers. Hence, fluorescence radiation is unsuitable to determine the response function of the apparatus.

As an alternative and more general approach to both $\gamma$ rays or natural $\mathrm{x}$ rays, we thus propose to profit from recent developments in exotic-atoms research and in heavy-ions sources, and to use two- and three-body systems as photon emitters in the few $\mathrm{keV}$ range. In contrast to $\gamma$ rays, electronic, muonic, and pionic atoms would provide a dense set of lines that can be supplemented by an even denser set if one can use antiprotonic atoms with 
beam intensities comparable to LEAR at CERN [8]. Our program consists first in doing relative energy measurements of transitions in one- and two-electron ions, emitted by the plasma of electron-cyclotron ion sources (ECRIS), of circular transitions in fully stripped pionic atoms and of $\mathrm{x}$ rays from solid fluorescence targets. Modern, commercial, permanent-magnet ECRIS are small and relatively economical to operate, and could be available in a large number of places to provide reference lines.

This relative energy scale will then be tied to a few, very bright lines, the energy of which will be measured absolutely, with either a double-flat crystal instrument, or a backscattering spectrometer as in [2] obtained from an electron-cyclotron ion trap (ECRIT), a device derived from the ECRIS, and optimized for increasing the trapping time of the ions [9], and thus the production of $x$ rays from highly charged ions. Intense M1 radiation from the $1 s 2 s^{1} S_{0} \rightarrow 1 s^{2}$ transition in heliumlike argon has been observed both in conventional ECRIS [10] and in the first run of the PSI ECRIT in 2002 [11]. The ions energy in such a device ranges from $\approx 0.5$ to $6 \mathrm{eV}$ depending on the injected RF power [12], giving rise to a Doppler broadening in the 5 to $18 \mathrm{ppm}$, i.e., 0.07 to $0.28 \mathrm{eV}$ for Ar. This allows for measurements well below $1 \mathrm{ppm}$, and corresponds also to the expected accuracy (typically $1 \mathrm{meV}$ ) of theory for one electron ions in this range of $Z$. Different energy ranges and diffraction orders can be connected by exploiting the yrast structure of the exotic-atom cascade, which leads to a strong population of circular states, thus favoring transitions with a change of the principal quantum number $n$ by 1 . Hence, successive transitions in the same atom connect different energy scales in ratios $\approx\left[1 /(n+1)^{2}-1 / n^{2}\right] /\left[1 / n^{2}-1 /(n-1)^{2}\right]$, which are roughly of the order of 0.5 for $n$ around 5. For instance, $\pi$ Ne provides the photon energies of $2.7,4.5$, and $8.3 \mathrm{keV}$ corresponding, respectively, to the $7 \rightarrow 6,6 \rightarrow 5$, and $5 \rightarrow 4$ transitions. With such a comparison method one can transfer energy standards between orders without problems due to changes in index of refraction.

The present program has several advantages. First, once a set of lines has been measured and compared to a given accuracy to quantum-electrodynamics (QED) calculations, other lines from the same source or from neighboring elements can be used as standards without the need of a direct measurement. Transition energies of these simple systems can probably be calculated nowadays from first principle to better than $1 \mathrm{meV}$, benefiting from high-accuracy tests of QED in hydrogen [13]. This is even true for hadronic atoms, if one uses circular transitions, which are not affected by strong interaction. Only particle masses (very well known except for pion), the fine-structure constant and the Rydberg constant are needed. Over time the precision of the calculation can be improved by systematically including contributions from higher-order Feynman diagrams. The quality of the cal- culation can be checked by comparing to the directly measured lines energies as well as to all the relatively measured ones which are directly connected to the direct measurements.

Second, the natural linewidths of these transitions, while not as small as $\gamma$-ray line, are 3 orders of magnitude narrower than the ones of multielectronic systems.

Finally, the combination of the exotic-atom and electronic $\mathrm{x}$-ray sources can provide a powerful tool for establishing high-quality x-ray energy standards. As an example, the $5 g \rightarrow 4 f$ transition in $\pi \mathrm{C}$, the Lyman- $\alpha$ in hydrogenlike $\mathrm{Cl}$, and the $\mathrm{K} \alpha$ fluorescence line in singly ionized $\mathrm{Ar}$, degenerate in energy by a few $\mathrm{eV}$, can be easily related by the method presented here.

In the present Letter, we present the measurement, with a crystal spectrometer of the characteristic $\mathrm{x}$ radiation from hydrogenlike pionic atoms. We use these transitions as energy standards for the energy determination of the transition energies in a complex electronic systems, the $\mathrm{K} \alpha_{1,2}$ transitions in Ti. In this way we intend to show that the pionic transition is in good agreement with a reasonably well measured $\mathrm{K} \alpha_{1,2}$ transition. Copper would have made a better case, but the present world average for the pion mass involve the $\mathrm{Cu} \mathrm{K} \alpha$ doublet. This issue will, however, be solved when the final value for the pion to muon mass ratio is released [14].

For this experiment we used the cyclotron trap II [15] attached to the $\pi \mathrm{E} 5$ pion line at the Paul Scherrer Institute (PSI, Switzerland). In this device a $112 \mathrm{MeV} / c$ pion beam is decelerated in a magnetic field using a suitable set of degrader foils. Such a setup allows the use of dilute targets such as gases. Typically $4 \times$ $10^{8} \pi^{-} / \mathrm{s}$ are injected in the trap for $1 \mathrm{~mA}$ proton current. The target consists of a cylinder of $60 \mathrm{~mm}$ diameter and $26 \mathrm{~cm}$ length, with $50 \mu \mathrm{m}$-thick kapton walls. The pressure in the target was around 1 bar, leading to typically $1.7 \times 10^{6} \pi^{-} / \mathrm{s}$ stops in the gas.

For light elements $(Z \leq 10)$ the cascade that follows leads quickly to the formation of an hydrogenlike exotic atom in a circular state. All the electrons are ejected by Auger effect in the early stage of the cascade, in a process similar to internal conversion in nuclei, because of the large mass of the pion $\left(\approx 273 \times m_{e^{-}}\right)$. Accordingly transition energies are 273 times larger than electronic ones between states of identical quantum numbers. Because the atoms are formed in a low-pressure gas the time it takes to recapture electrons from molecules in the gas is much longer than the pionic atom lifetime, hence, the exotic atoms stay in a hydrogenlike state for the rest of the atomic cascade. It has been shown that less than $2 \%$ of the $x$ ray observed are affected by the presence of an extra electron [16]. When using solid targets the undefined status of the electron shell is the principal limitation in high-precision experiments using exotic-atom $x$ rays [17].

$X$ rays emitted by the exotic atoms at the center of the trap are analyzed using a Johann-type Bragg 
spectrometer that was developed for applications requiring very high luminosity and excellent resolution in noisy environments, in the 1.7 to $10 \mathrm{keV}$ range. It was equipped with a spherically bent $\mathrm{Si}(220)$ crystal having a radius of curvature of $2.9854 \mathrm{~m}$. A detailed description of the apparatus is given in $[8,16,18]$.

For this proof-of-principle measurement we relate the energy of the $6 h \rightarrow 5 g$ transition in pionic neon to energy of the $\mathrm{K} \alpha_{1,2}$ transitions of Ti. The Ti K $\alpha_{1}$ differs only by a fraction of an $\mathrm{eV}$ from the strong $6 h \rightarrow 5 g$ transition in $\pi^{20} \mathrm{Ne}$. Hence, the measurement could be performed by exchanging only the neon-filled target cell with a $30 \times$ $20 \mathrm{~mm}^{2}$ plate of metallic Ti, without any other change of the experimental setup. The fluorescence $\mathrm{x}$ rays were excited by means of an $\mathrm{x}$-ray tube with a $\mathrm{Cr}$ anode. The consecutively recorded $\pi \mathrm{Ne}$ and $\mathrm{Ti}$ spectra are shown in Fig. 1.

The pionic transition energies are calculated from the world average pion mass $m_{\pi}=139.57018 \pm$ $0.00035 \mathrm{MeV}$ as given by the particle data group [19] and fundamental constants [20] with the Klein-Gordon equation for a spherical nuclear charge distribution (to improve numerical stability although direct effect on energy is small). They include the Uehling potential for vacuum polarization to all order, the Källèn and Sabry as well as the Wichman and Kroll correction, and include nuclear recoil and relativistic recoil. The nuclear masses for ${ }^{20} \mathrm{Ne}$ and ${ }^{22} \mathrm{Ne}$ are deduced from atomic masses in $[21,22]$, respectively, by subtracting the mass of the ten missing electrons. These energies can be calculated with

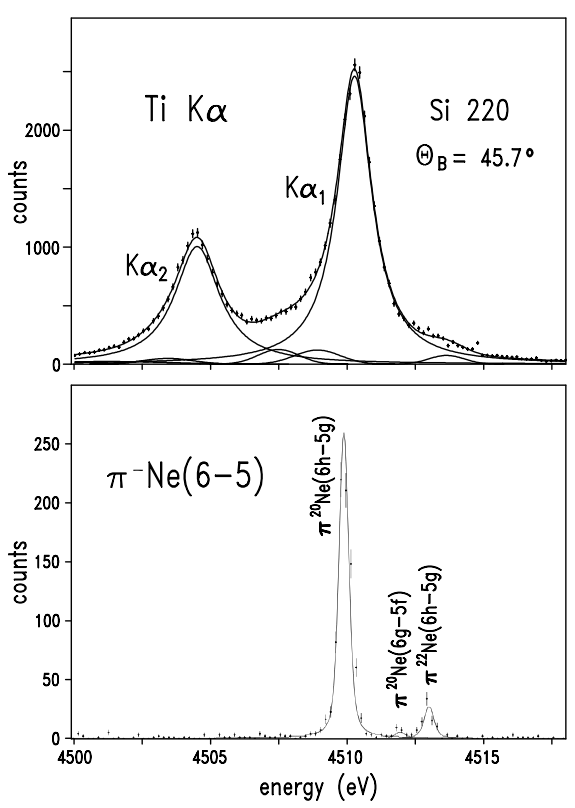

FIG. 1. Upper : Ti Ka doublet fitted by a sum of six Voigt functions. Lower : $\pi \mathrm{Ne} 6 \rightarrow 5$ transitions showing fine-structure and isotopic effects. Comparison of the two spectra demonstrates the energy coincidence of the $\mathrm{Ti} \mathrm{K} \alpha_{1}$ and of the $\pi^{20} \mathrm{Ne} 6 h \rightarrow 5 g$ lines. high precision since the strong interaction plays no role for such high-lying circular states. We obtain 4509.894 and $4512.948 \mathrm{eV}$ for the $6 h \rightarrow 5 g$ transition in $\pi^{22} \mathrm{Ne}$ and $\pi^{20} \mathrm{Ne}$, respectively. There is an uncertainty of $11 \mathrm{meV}$ on these energy values which originates exclusively from the pion mass.

The natural width of the $\pi \mathrm{Ne} 6 h \rightarrow 5 g$ transition is $12 \mathrm{meV}$. The fact that the calibration line is almost a $\delta$ function allows for precise determination of the spectrometer response function. The measured instrumental response function width of $440 \pm 20 \mathrm{meV}$ is close to the theoretical limit of $330 \mathrm{meV}$ as predicted from the Monte Carlo simulations for the chosen geometry. The $\pi \mathrm{Ne}$ line shape is fitted sufficiently well by using Gaussian line profiles.

To deduce the Ti K $\alpha$ line shape we fitted a sum of six Voigt profiles, following [18] using the Gaussian response function obtained from the $\pi \mathrm{Ne}$ spectrum. The peak positions, $\mathrm{K} \alpha_{1,2}^{0}$, are obtained from the zeros of the derivative of the fitted function. The spectrometer dispersion, necessary to transform the position information of the detector to energy, is obtained in a self-consistent way from the $\pi \mathrm{Ne}$ spectrum itself (Fig. 1). For the very small energy difference, for the case of $\pi \mathrm{Ne} 6 \rightarrow 5$ and $\mathrm{Ti}$ $\mathrm{K} \alpha$, the energy-dependent corrections originating from the imaging properties of crystal spectrometer (refraction index, crystal bending and size, rocking curve) are almost invariable and cancel out.

The results are displayed in Table I, together with presently known values. The experimental error in the energy of the $\mathrm{K} \alpha_{1}$ line is practically given by the statistical uncertainty on the $\mathrm{K} \alpha_{1}$ and $\pi^{20} \mathrm{Ne} 6 h \rightarrow 5 g$ lines. In the case of the $\mathrm{K} \alpha_{2}$ line the error is dominated by the uncertainty on the dispersion value, which is due to the limited statistics of the $\pi^{22} \mathrm{Ne} 6 h \rightarrow 5 g$ transition. Both contributions could be drastically reduced by increasing the statistics of the measurement.

Narrow transitions from exotic atoms allow one to characterize very precisely the response function of a curved crystal setup. For the described experiment the accuracy of the extracted natural widths of the electronic systems (Table I) reaches the one obtained with ultimate resolution devices such as double-flat crystal spectrometers $[7,23]$.

In the present Letter we have demonstrated that narrow lines from hydrogenlike pionic atoms are able to serve as energy standards in the few $\mathrm{keV}$ range. By using this method the energy uncertainty is limited primarily by the knowledge of the charged pion mass, whenever transitions close in energy can be found. We have proved that a hydrogenic pionic line, the energy of which has been calculated from QED, can be used to establish the energy of a previously well-measured line with comparable accuracy. A research program is underway to improve on the precision of the pion mass to the order of about $1 \mathrm{ppm}$ [14]. An alternative is to use muonic transitions as the 
TABLE I. Peak position energies and natural widths of the $\mathrm{K} \alpha_{1,2}$ transitions in metallic Ti in comparison with previous measurements (in $\mathrm{eV}$ ). For this work the errors on the energies from the experiment (first parenthesis) and from the calibration standard, i.e., the uncertainty of the pion mass (second parenthesis), are given separately.

\begin{tabular}{cccccc}
\hline \hline Element & Line & Energy(this work) & Energy (Refs. [3]) & Width(this work) & Width \\
\hline \multirow{2}{*}{$\mathrm{Ti}$} & $\mathrm{K} \alpha_{1}^{0}$ & $4510.903(19)(11)$ & $4510.869(49)$ & $1.6(1)$ & $1.5(3)^{\mathrm{a}}$ \\
& $\mathrm{K} \alpha_{2}^{0}$ & $4504.942(40)(11)$ & $4504.887(49)$ & $2.1(1)$ & $2.1(4)^{\mathrm{a}}$ \\
\hline \hline
\end{tabular}

${ }^{\mathrm{a}}$ Ref. [23]

muon mass is known to 0.05 ppm [19]. However, intensities achievable are about 2 orders of magnitude smaller than in the case of pionic atoms. Much higher intensity will be available from $\mathrm{x}$-ray sources such as the superconducting electron-cyclotron ion trap (ECRIT) developed at the Paul Scherrer Institute, in which few-electron atoms up to hydrogenlike systems are produced [9]. Such electronic two-body systems will be used as calculable energy standards in the same way as exotic atoms.

In a separate experiment we have recently used the technique presented here to measure the energy of the Sc $\mathrm{K} \alpha$ lines, which are known only from interpolation [24], with an accuracy improved by a factor of 12 [25].

By using x-ray lines, both from hydrogenlike exotic and electronic atoms a relative energy scale is established that can easily range up to $30 \mathrm{keV}$. Around 60 lines will be available if electronic, pionic and muonic atoms are used, hundred more if antiprotonic atoms are available. The energy of these lines will depend only on the fine-structure constant $\alpha$, and on the pion, muon and electron mass, and they can also be connected to low-energy $\gamma$-ray standards.

*Email address: paul.indelicato@ spectro.jussieu.fr Electronic address: http://dirac.spectro.jussieu.fr 'Laboratoire Kastler Brossel is Unité Mixte de Recherche du CNRS no. 8552, of the Physics Department of École Normale Supérieure and Université Pierre et Marie Curie.

[1] J. Härtwig, S. Grosswig, P. Becker, and D. Windisch, Phys. Status Solidi (a) 125, 79 (1991).

[2] Y.V. Shvyd'ko et al., Phys. Rev. Lett. 85, 495 (2000).

[3] R. Deslattes, E. Kessler, Jr., P. Indelicato, L. de Billy, E. Lindroth, and J. Anton, Rev. Mod. Phys. 75, 35 (2003); http: //www. physics. nist. gov/ Phys RefData / Xray Trans / index.html
[4] R. D. Deslattes, P. L. Cowan, and R.E. LaVilla, AIP Conf. Proc. No. 94 (AIP, New York, 1982), p. 100.

[5] R. D. Deslattes, R. E. LaVilla, P. L. Cowan, and A. Henins, Phys. Rev. A 27, 923 (1983).

[6] P. Indelicato, S. Boucard, and E. Lindroth, Eur. Phys. J. D 3, 29 (1998).

[7] J. Kawai, E. Nakamura, Y. Nihei, K. Fujisawa, and Y. Ghoshi, Spectrochim. Acta, Pt. B 45, 463 (1990).

[8] D. Gotta et al., Nucl. Phys. A660, 283 (1999).

[9] S. Biri, L. Simons, and D. Hitz, Rev. Sci. Instrum. 71, 1116 (2000).

[10] G. Douysset, H. Khodja, A. Girard, and J. P. Briand, Phys. Rev. E 61, 3015 (2000).

[11] D. F. Anagnostopoulos et al., Nucl. Instrum. Methods Phys. Res., Sect. B 205, 9 (2003).

[12] C. Bernard, Ph.D. thesis, Université Claude Bernard, 1996.

[13] B. de Beauvoir, C. Schwob, O. Acef, L. Jozefowski, L. Hilico, F. Nez, L. Julien, A. Clairon, and F. Biraben, Eur. Phys. J. D 12, 61 (2000).

[14] N. Nelms et al., Nucl. Instrum. Methods Phys. Res., Sect. A 477, 461 (2002).

[15] L. Simons, Hyperfine Interact. 81, 253 (1993).

[16] S. Lenz et al., Phys. Lett. B 416, 50 (1998).

[17] B. Jeckelmann, P. Goudsmit, and H. Leisi, Phys. Lett. B 335, 326 (1994).

[18] D. F. Anagnostopoulos, R. Sharon, D. Gotta, and M. Deutsch, Phys. Rev. A 60, 2018 (1999).

[19] Particle Data Group, D. Groom et al., Eur. Phys. J. C 15, 1 (2000); http://pdg.lbl.gov

[20] P. J. Mohr and B. N. Taylor, Rev. Mod. Phys. 72, 351 (2000).

[21] F. DiFilippo, V. Natarajan, K. R. Boyce, and D. E. Pritchard, Phys. Rev. Lett. 73, 1481 (1994).

[22] G. Audi and A. Wapstra, Nucl. Phys. A595, 409 (1995).

[23] S. I. Salem and P. L. Lee, At. Data Nucl. Data Tables 18, 233 (1976).

[24] J. Bearden, Rev. Mod. Phys. 39, 78 (1967).

[25] D. Anagnostopoulos, D. Gotta, P. Indelicato, and L. Simons (to be published). 